\title{
Generalised Holography Meets Coherent Diffractive Imaging
}

\author{
A. J. D’Alfonso ${ }^{1}$,A. J. Morgan ${ }^{1}$, A. W. Yan ${ }^{1}$, P. Wang ${ }^{2}$, H. Sawada ${ }^{3}$, A. I. Kirkland ${ }^{4}$ and L. J. Allen ${ }^{1}$ \\ ${ }^{1}$ School of Physics, University of Melbourne, Parkville, Victoria 3010, Australia \\ ${ }^{2}$ National Laboratory of Solid State Microstructures and Department of Materials Science and \\ Engineering, Nanjing University, Nanjing 210093, People's Republic of China \\ 3.JEOL Ltd, 1-2 Musashino 3-Chome, Akishima, Tokyo 196, Japan \\ ${ }^{4}$.Department of Materials, University of Oxford, Parks Road, Oxford OX1 3PH, UK
}

Coherent diffractive imaging (CDI) is an imaging technique that seeks to restore the exit-surface wave from intensity measurements, either in the far field diffraction plane or the Fresnel diffraction region. To date numerous strategies have been proposed to complete this restoration, the most successful being the original scheme proposed by Gerchberg and Saxton and its subsequent modifications [1]. These schemes all involve the iterative refinement of a trial wave function using a-priori known information about the sample in conjunction with the measured amplitude to constrain the proposed exit surface wave function phase. These approaches use the self interference of the exit surface wave to transform the reconstruction process into a nonsmooth, nonconvex nonlinear optimization problem and as such are plagued by uniqueness issues. Concurrent to developments in CDI have been developments to Fourier Holography [2]. In Fourier holography the measured interference between a reference wave and the unknown scattered wave is used to deduce the complex exit surface wave. Under special circumstances where the reference wave has a particular structure (i.e. a pinhole or uniformly redundant array in the object plane), the inversion from the diffraction measurement is direct and a unique solution is obtained.

Using a generalized approach to Fourier Holography [3,4] allows the autocorrelation of the exit surface wave, which is the inverse Fourier transform of the diffraction pattern, to be analysed and a set of linear equations may be constructed allowing the direct reconstruction of the exit surface wave. Furthermore, as the co-efficient matrix has a Hankel plus Toeplitz-like structure the solution to the matrix equation may be solved efficiently using the conjugate gradients least squares method implemented using fast Fourier transforms [4].

Using a scanning transmission electron microscopy (STEM) probe we have applied this generalized holographic CDI approach to achieve an atomic resolution reconstruction of a specimen transmission function from a cerium dioxide nanoparticle from a single far field intensity measurement. The diffraction pattern (also known as a Ronchigram or Gabor Hologram) formed using a defocused STEM probe is shown in Fig. 1(a). A high angle annular dark field (HAADF) image of the edge of the nanoparticle along with the computed illumination amplitude and its position with respect to the specimen for the diffraction pattern in Fig 1(a) is shown in Fig. 1(b). The phase of the specimen transmission function, reconstructed using the generalized holographic approach, for two independent Ronchigrams is overlaid onto the HAADF image in Fig. 1(c).

Using a linear frame work to solve the holographic CDI problem affords deep insight into proposed CDI methods. One such method is ptychography which uses multiple measurements in the diffraction plane concurrently to reconstruct the specimen transmission function [5]. Unlike conventional CDI where a compact support in the object plane is typically required, ptychography uses consistency in the common area between overlapping probe positions as an object plane constraint, reducing the quantity of a-priori 
knowledge about the specimen that is needed, while also over-determining the reconstruction process. We applied the principles of ptychography within the context of generalized holography to reconstruct the transmission function, at atomic resolution, of a second cerium dioxide nanoparticle [6]. The transmission function is shown in Fig. 2, and when compared to Fig. 1 demonstrates the robust nature of the technique.

\section{References:}

[1] RW Gerchberg and W O Saxton, Optik, 35(1972), p. 237.

[2] S Eisebitt, et al., Nature, 432 (2004), p. 885.

[3] AV Martin and L. J. Allen, 281 (2008), p. 5114.

[4] AJ D’Alfonso, et al., Physical Review A, 85 (2012), 013816.

[5] AM Maiden and J. M. Rodenburg, Ultramicroscopy, 109 (2009), p. 1256.

[6] AJ D’Alfonso et al., Physical Review B, in press, (2014).

[7] This research was supported under the Australian Research Council's Discovery Projects funding scheme (Project No. DP1096025) and its DECRA funding scheme (Project No. DE130100739) and by the EPSRC (Grant Nos. EP/E034055/1 and EP/F048009/1). The microscope was developed under the CREST R005 project, supported by the Japan Science and Technology Agency (Project leader: Kunio Takayanagi).
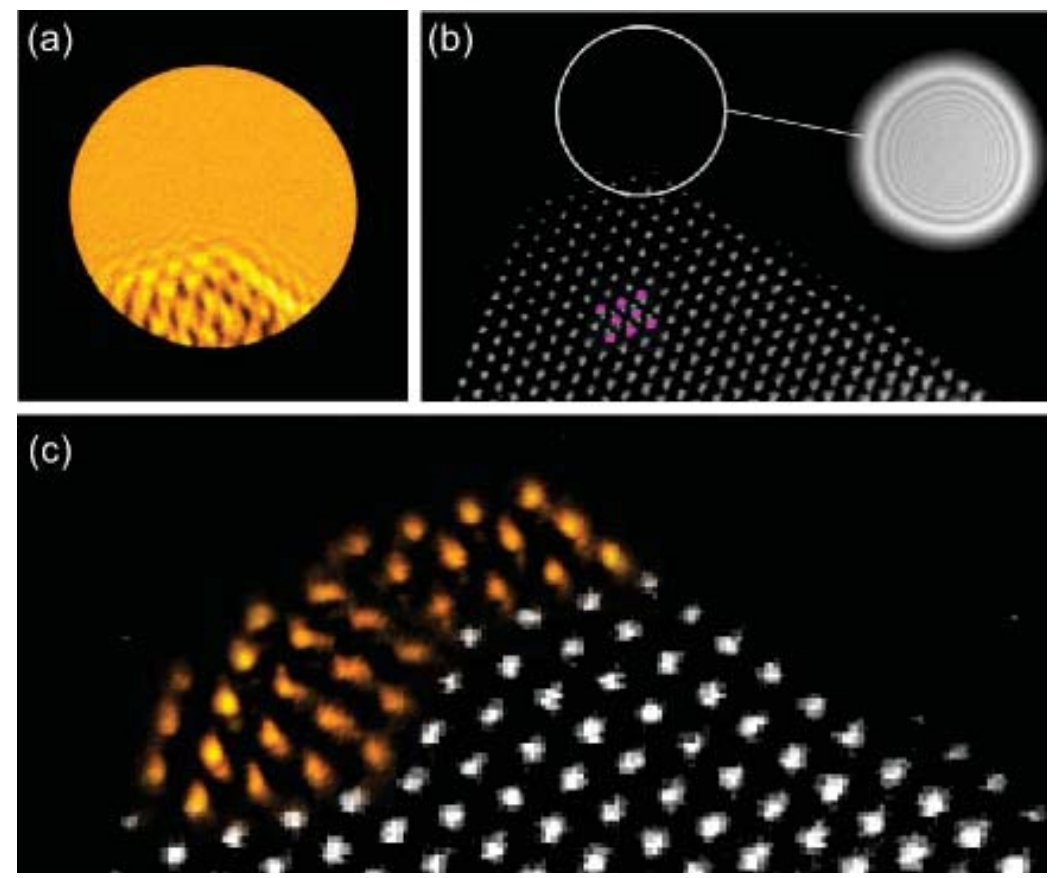

Figure 1. (a) The diffraction pattern obtained using a defocused STEM probe of a $\mathrm{CeO}_{2}$ nanoparticle. (b) HAADF image with the computed probe amplitude. The position of the probe for the diffraction pattern in (a) is indicated by the circle over the top right corner in (b). The projected structure of $\mathrm{CeO}_{2}$ is indicated. The larger pink circles indicate cerium columns and the smaller green circles indicate oxygen columns. (c) Two independent reconstructions of the phase of the transmission function are overlaid onto the HAADF image of the specimen. The $300 \mathrm{keV}$ probe was formed using a $24 \mathrm{mrad}$ aperture.

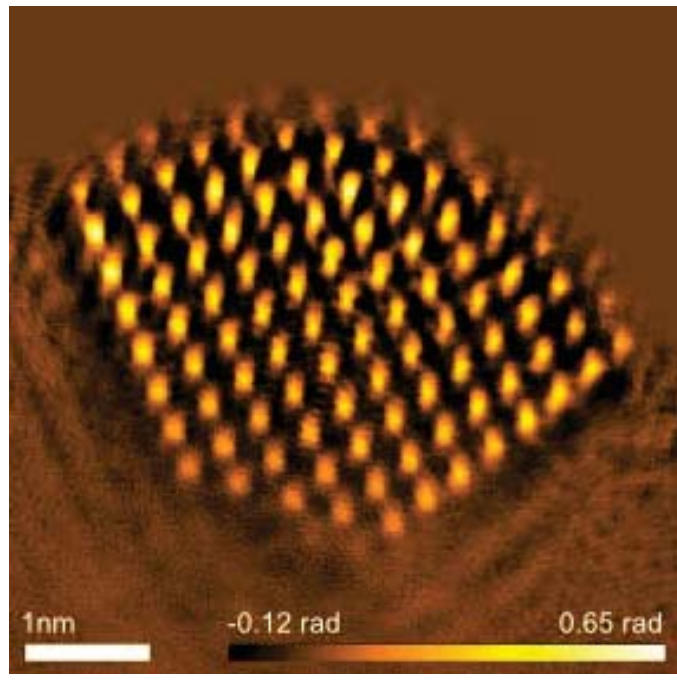

Figure 2. The phase of the transmission function of $\mathrm{CeO}_{2}$ specimen reconstructed using the generalized holography method in Ref. [6]. The diffraction data was recorded using a $300 \mathrm{keV}$ probe formed using a $24 \mathrm{mrad}$ aperture. 